\title{
Justificación teórica de la reparación de un edificio de concreto con valor histórico
}

\author{
J. A. Briceño-Mena ${ }^{1}$, P. Castro-Borges ${ }^{1 *}$ \\ *Autor de Contacto: pcastro@cinvestav.mx; pcb1963@gmail.com \\ ${ }^{1}$ Departamento de Física Aplicada, Centro de Investigación y de Estudios Avanzados del Instituto Politécnico \\ Nacional, Unidad Mérida. Km 6 Antigua Carretera a Progreso, Mérida, Yucatán, México
}

\begin{abstract}
RESUMEN
Se justificó teóricamente, y se llevó a cabo, la reparación de un edificio de concreto reforzado con valor histórico (casi 70 años), para rescatarlo ante un cambio de uso. La evaluación por durabilidad arrojó información para que el edificio pudiese ser rescatado en un $80 \%$. Para lograrlo, se utilizaron estrategias que permitieron una mejor utilización de los recursos materiales, equipamiento y mano de obra en la reparación de muros, columnas, losas y trabes. Una de las estrategias fue usar el factor de seguridad teórico para calcular la profundidad de reparación sin consecuencias estructurales, pero cumpliendo la finalidad de controlar la carbonatación del concreto, y manteniendo la predicción deseada de vida de servicio. Como ejemplo se ilustra el caso de las columnas.
\end{abstract}

Palabras clave: Restauración; reparación; concreto reforzado; durabilidad; factor de seguridad

\section{RESUMO}

Teoricamente justificou e realizou a reparação de um edifício de betão armado com valor histórico (quase 70 anos), para o resgatar de uma mudança de uso. A avaliação da durabilidade rendeu informações para que o prédio pudesse ser resgatado em $80 \%$. Para tal, foram utilizadas estratégias que permitiram um melhor aproveitamento dos recursos materiais, equipamentos e mão-de-obra na reparação de paredes, pilares, lajes e vigas. Uma das estratégias foi utilizar o fator de segurança teórico para calcular a profundidade de reparo sem consequências estruturais, mas cumprindo o propósito de controlar a carbonatação do concreto, e manter a previsão de vida útil desejada. Como exemplo, o caso das colunas é ilustrado.

Palavras-chave: Restauração; reparar; concreto reforçado; durabilidade; fator de segurança

\begin{abstract}
Theoretically justified, and carried out, the repair of a reinforced concrete building with historical value (almost 70 years), to rescue it from a change of use. The durability evaluation yielded information so that the building could be rescued by $80 \%$. To achieve this, strategies were used
\end{abstract}


that allowed a better use of material resources, equipment, and labor in the repair of walls, columns, slabs, and beams. One of the strategies was to use the theoretical safety factor to calculate the repair depth without structural consequences but fulfilling the purpose of controlling the carbonation of the concrete and maintaining the desired prediction of service life. As an example, the case of columns is illustrated.

Keywords: Restoration; repair; reinforced concrete; durability; security factor

\section{INTRODUCCIÓN}

Uno de los problemas comunes en las reparaciones del concreto estriba en el uso erróneo de las especificaciones estructurales para conocer la profundidad a la que deben realizarse, cuando se enfrentan problemas por cloruros o carbonatación. Ello trae como consecuencia un uso poco optimizado de la mano de obra, equipamiento y materiales que conlleva, no sólo a reparaciones equivocadas y el retorno del problema, sino también una falta de sustentabilidad en todo el proceso. En el presente trabajo se ilustra, para el caso de las columnas, como fue utilizado el factor de seguridad (FS) para la reparación y rehabilitación de un edificio de concreto reforzado en estado de abandono, ubicado en el centro histórico de Campeche (Figura 1). Este edificio con un espacio interno de más de $600 \mathrm{~m}^{2}$ y una altura aproximada de $20 \mathrm{~m}$, y una estructura a base de marcos, suponía una demolición y posterior construcción de uno nuevo de 4 niveles. Sin embargo, este plan fue reconsiderado debido a que el edificio en cuestión tenía una edad aproximada de 70 años y se encontraba en el centro histórico de la ciudad de Campeche, Campeche, México, declarado patrimonio de la humanidad en el año 1999 por la Organización de las Naciones Unidas para la Educación, la Ciencia y la Cultura. Dicho lugar se encuentra protegido por autoridades federales como el Instituto Nacional de Bellas Artes y Literatura (INBAL) y el Instituto Nacional de Antropología e Historia (INAH). Por lo tanto, más que un proceso de demolición se tenía que considerar una estrategia de reparación y rehabilitación que cumpliera con las condiciones establecidas por las autoridades federales, donde se preservara lo más posible de la estructura $(80 \%)$. Por tal motivo, se realizó un estudio extenso sobre la durabilidad del inmueble. Como consecuencia, se definieron las partes que se mantendrían en pie previo a una intervención que implicaría reparación, rehabilitación y/o refuerzo de los distintos elementos estructurales, evitando los procesos de demolición iniciales y acoplándose a las condiciones requeridas. Se planteó adosar una estructura nueva de manera interna al edificio ya reparado y rehabilitado, manteniendo así su arquitectura exterior y rescatando zonas interiores. 


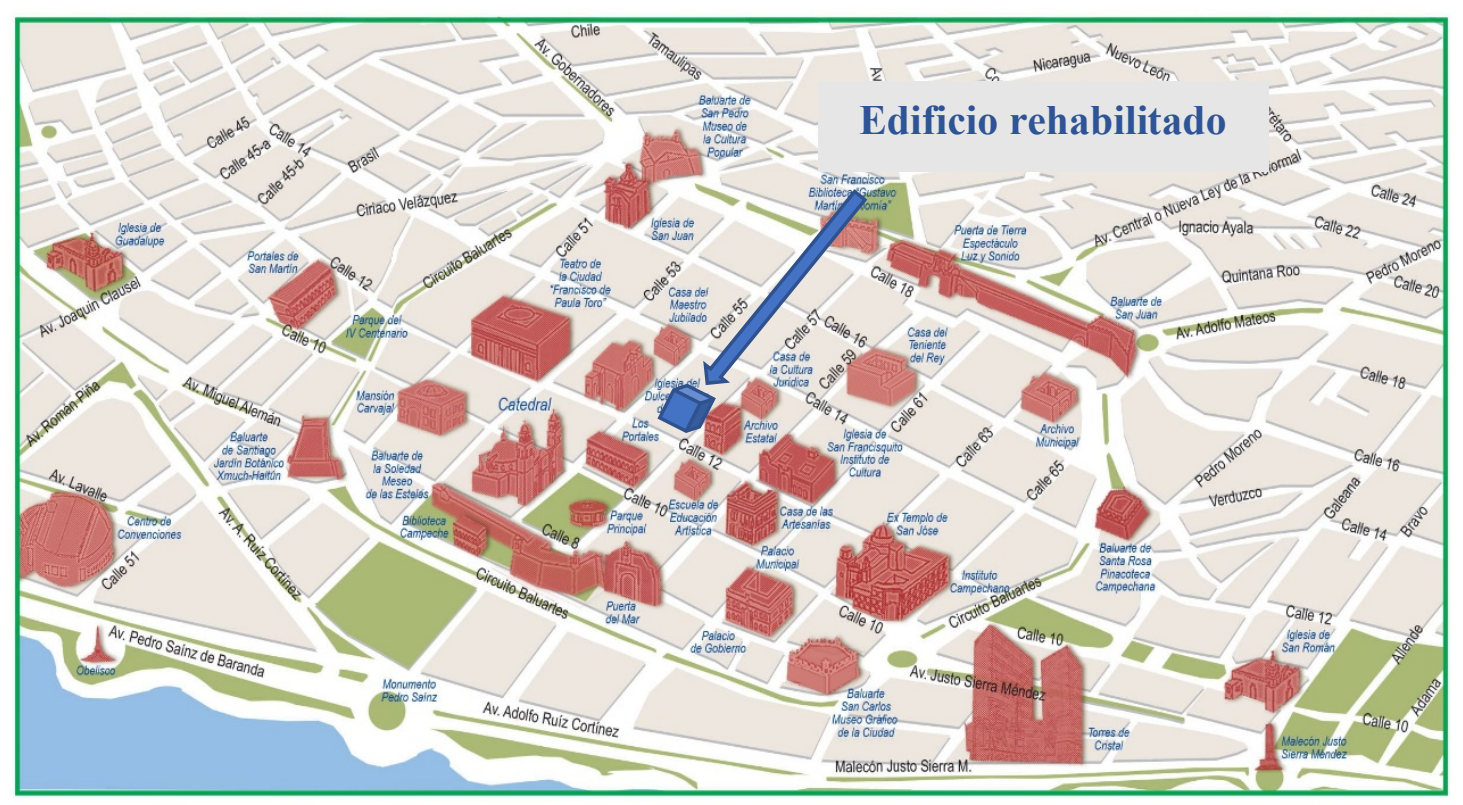

Figura. 1. Ubicación de la estructura dentro de la zona protegida de Campeche ${ }^{1}$

La estructura en cuestión tuvo como función primaria la de ser un cinema, posteriormente funcionó como un estacionamiento y finalmente fue abandonada. Estos cambios de uso y el abandono fueron factores que potencializaron los daños, como se observa parcialmente en las fotos de la Figura 2.
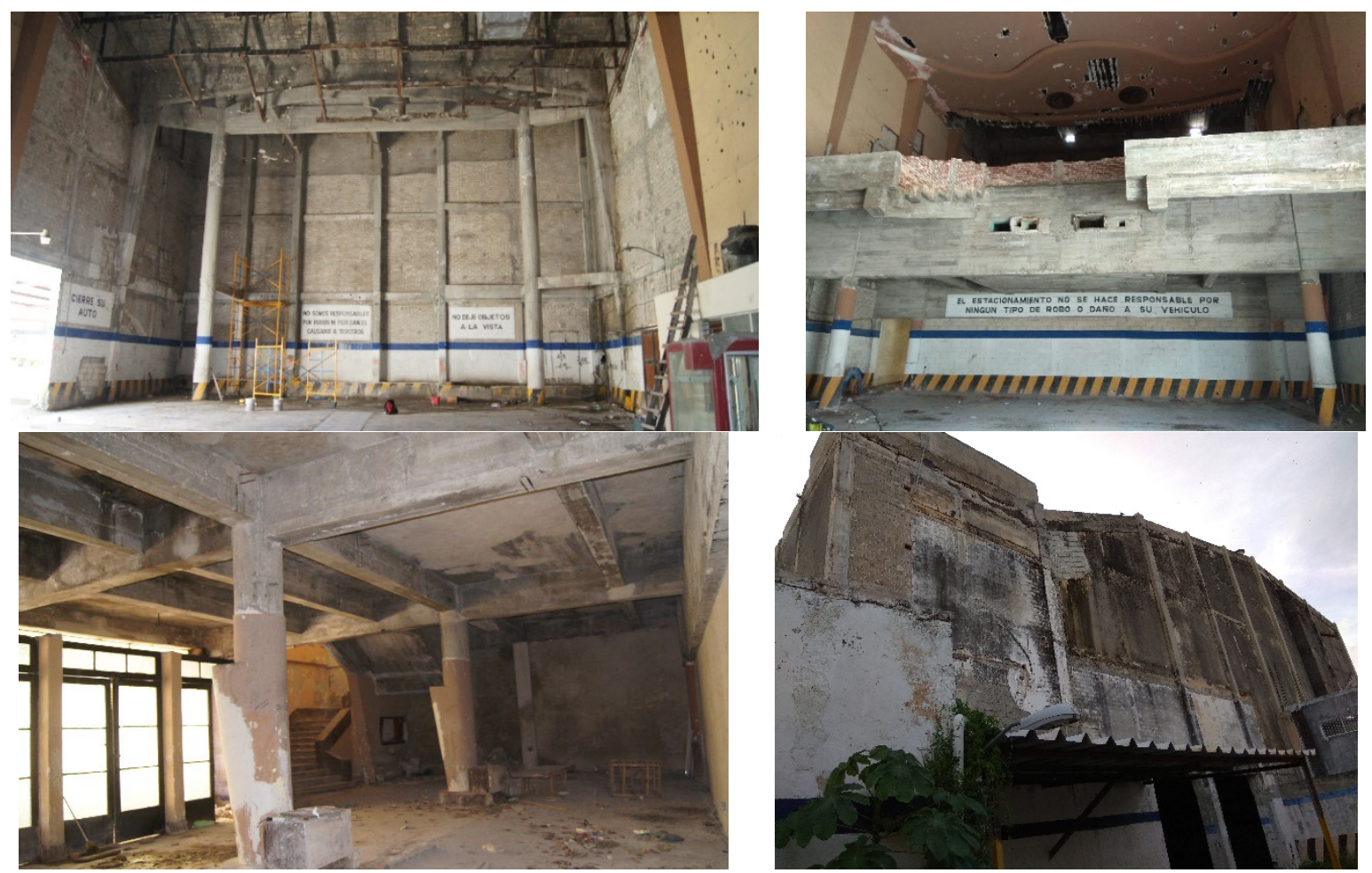

Figura. 2. Estado en que se encontraba la estructura antes de ser intervenida

${ }^{1}$ https://programadestinosmexico.com/descubre-mexico/mapas/mapas-de-ciudad-de-campeche.html 


\section{INSPECCIÓN DE DAÑOS}

Antes de realizar cualquier intervención en la estructura, fue necesario realizar una inspección de los daños, basada en la normativa mexicana [1], [2], para poder establecer las estrategias de reparación. Dichas inspecciones ya han sido publicadas [3] y pueden consultarse en la literatura para más detalle. Estas inspecciones tuvieron 3 ejes principales:

- Visual, para poder identificar las zonas con daños que requirieran una intervención mayor y posibles causantes de problemas patológicos como son zonas de humedad, así como desprendimientos, agrietamientos, etc.

- Mecánica, para conocer el estado del concreto como es su resistencia mecánica y poder establecer la resistencia del nuevo concreto a usar.

- Electroquímica, para conocer el estado del acero, que tan dañado podría estar por los problemas patológicos.

\section{ESTRATEGIAS DE REPARACIÓN}

Después de la inspección realizada, se pudo constatar que los elementos estructurales y arquitectónicos podrían ser intervenidos para su consolidación con la nueva estructura a construirse al interior del edificio como parte de su cambio de uso. Todas las estrategias de reparación fueron establecidas para poder brindar a la estructura una nueva vida de servicio y una durabilidad contra los problemas patológicos actuales y futuros a los que pueda verse sometida, tal como lo establece la normativa mexicana [4]. A su vez, estas estrategias se basaron en experiencias previas y en la literatura como es el manual Rehabilitar [5]. El fin de estas reparaciones/rehabilitaciones fue el de retirar el concreto dañado o que presentara algún problema patológico como carbonatación, así como asegurar la estabilidad estructural con el recálculo y restructuración de la cantidad de acero necesaria en los elementos, además de asegurar el recubrimiento adecuado según los requerimientos estructurales. La Figura 3 muestra procesos de deterioro de la estructura. Para delimitar las estrategias de reparación era necesario establecer los tipos de reparación a utilizar:
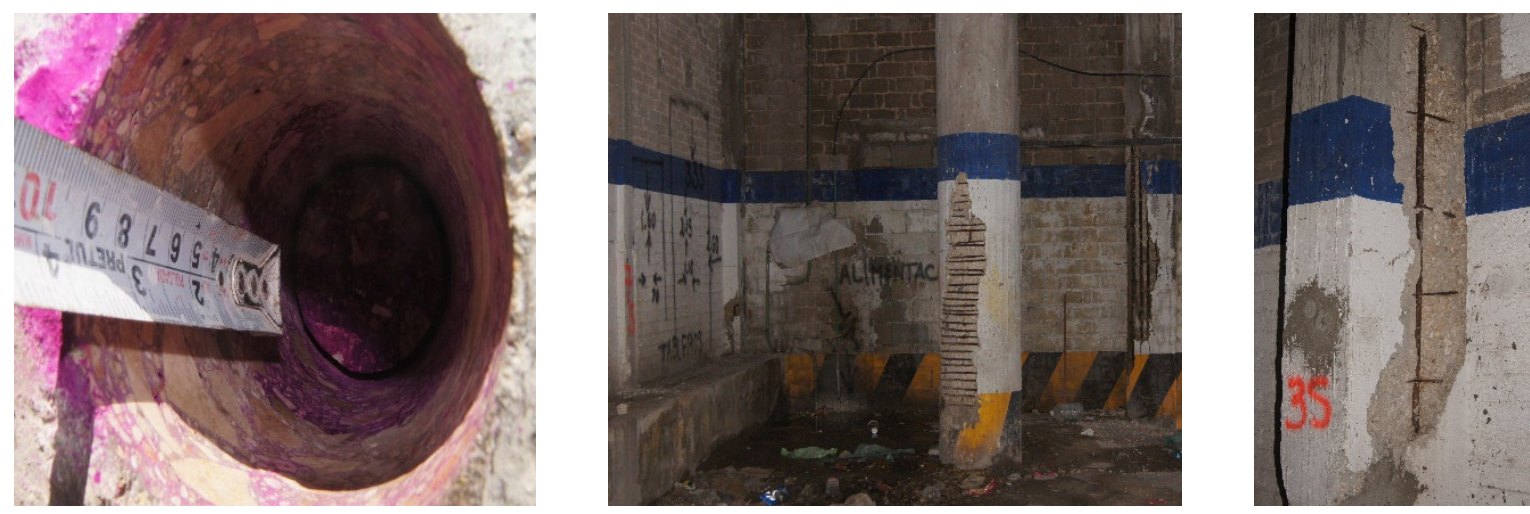

Figura. 3. Carbonatación y desprendimiento del concreto

\subsection{Reparación superficial localizada}

Esta reparación se refiere a aquellos elementos que presentaran daños en el concreto con poca profundidad y extensión en relación con las dimensiones del elemento estructural. Por lo anterior se estableció una reparación en zonas puntuales donde se aprecien agrietamientos a una profundidad no mayor de los $3 \mathrm{~cm}$ o antes de llegar al acero de refuerzo. 


\subsection{Reparación superficial uniforme}

Esta estrategia de reparación es parecida a la reparación superficial localizada ya que se realizará a una profundidad no mayor de $\operatorname{los} 2 \mathrm{~cm}$, o antes de llegar al acero de refuerzo, pero contemplando la reparación en una extensión relativamente grande de acuerdo con las dimensiones del elemento estructural, delimitando para los trabajos una extensión máxima sobre el elemento de $1.5 \mathrm{~m}$.

\subsection{Reparación profunda localizada}

Esta reparación se llevará a cabo en los elementos estructurales donde se hayan identificado daños con extensión grande en relación con las dimensiones del elemento estructural, siendo su profundidad mayor que $3 \mathrm{~cm}$, afectando el concreto detrás de las armaduras.

\subsection{Reparación profunda uniforme}

Al igual que en el apartado de reparación profunda localizada, la reparación se realiza a una profundidad más allá del acero. Sin embargo, al ser uniforme, se estableció la realización de la reparación por secciones máximas de $1.5 \mathrm{~m}$, debido a cuestiones estructurales.

\section{Planteamiento y uso del Factor de Seguridad en las reparaciones}

Las técnicas de reparación planteadas se realizaron con el fin de aplicar en los procesos constructivos herramientas que permitan evitar procesos innecesarios como la demolición, que traerían consigo la generación de escombros y basura y más aún en zonas importantes como lo es un patrimonio de la humanidad.

Como punto de partida inicial para realizar la reparación, fue importante analizar cada elemento individualmente, su comportamiento, su carga portante máxima, su estado, y posteriormente proyectarlo a un nivel general relacionándolo con todos los elementos estructurales, obteniendo así un comportamiento global. De esta forma, fue posible realizar la intervención considerando todos los factores de afectación posibles y minimizando daños colaterales del proceso de reparación. Esto a su vez conlleva a realizar una capacitación del personal a elaborar las reparaciones ya que, en la actualidad, tanto los obreros como los ingenieros, desconocen de estos temas lo que provoca un uso incorrecto de herramientas, materiales y equipo.

Si bien, una estrategia de reparación puede ser realizada por gente capacitada, siempre es importante tener en cuenta los factores de seguridad y riesgo a la hora de trabajar. En la literatura podemos encontrar información acerca de la Teoría de Seguridad [6] que, en general, consiste en relacionar el nivel de seguridad $(\mathrm{H})(1)$, definido como $\mathrm{H}=1$ - pf, donde pf es la probabilidad de falla. Este nivel de seguridad depende de todas las variables que intervienen en el diseño de la estructura y de cómo pueden variar los mismos. La ecuación general que rige la teoría de seguridad es:

$$
\mathrm{H} \geq \mathrm{H}^{\mathrm{n}}
$$

Donde: $\mathrm{H}^{\mathrm{n}}$ es el nivel de seguridad del diseño

$\mathrm{H}$ es el nivel de seguridad de obra.

En Ingeniería Civil, se puede entender como seguridad el evitar que la estructura sobrepase el estado límite permisible el cual es donde se considera que el comportamiento estructural es aceptable[7]. El factor de seguridad (FS) es aquel que relaciona la carga o solicitación (C) a 
aplicarse en la estructura con la capacidad máxima (R) que esta puede soportar, es decir, su resistencia máxima.

$F S=\frac{C}{R}$

Poniendo un ejemplo práctico fuera de la realidad, se puede entender el factor de seguridad de la siguiente manera: Supongamos que se tiene una columna, la cual fue diseñada para aguantar 6 toneladas de carga y en ella descansaran 2 vigas, y cada una de estas transferirá una carga de 2 toneladas a la columna. Por lo tanto, podemos apreciar que nuestro FS es de 1.5 (FS =6/4 = 1.5). Aunque en este ejemplo se trató como un tema simple, en la realidad es algo mucho más complejo. Se deben considerar diversos criterios como son las cargas vivas (las personas que utilizarán el edificio), las cargas muertas (mobiliario, por ejemplo), las cargas accidentales, así como factores ambientales.

Dicha teoría, fue llevada a la práctica en el proceso de reparación de la estructura descrita en este trabajo. Para ello se requirió el apoyo de manuales, normativa, literatura y experiencia propia para establecer con el mejor criterio la estrategia idónea por elemento. Como se comentó anteriormente, gracias a la inspección previa se pudo establecer el grado de daño y con ello la estrategia de reparación. Si bien se establecieron 4 posibles, en este trabajo solo discutiremos lo relacionado a la reparación profunda uniforme (véase Figura 4)
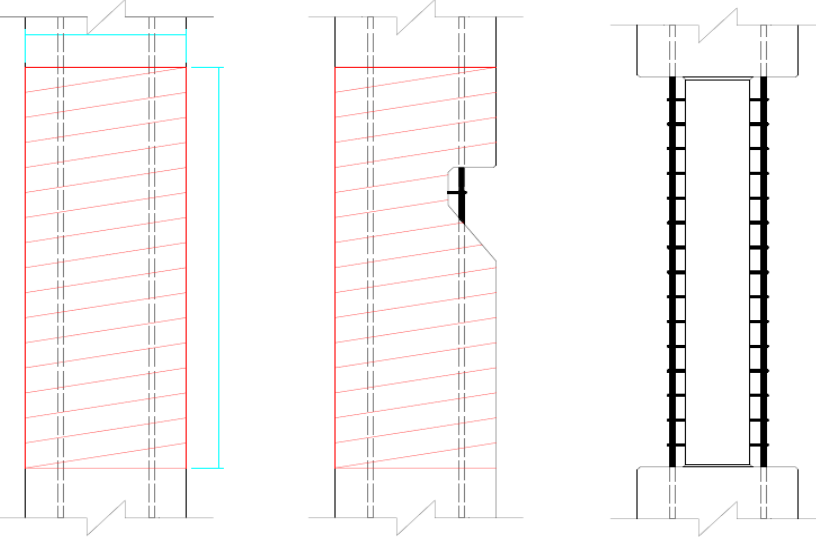

Figura. 4. Reparación profunda uniforme

Para poder llevar a cabo las reparaciones, fue necesario establecer el criterio con el cual se realizarían. En el caso de esta estructura tuvimos elementos de distintas geometrías y dimensiones por lo que se establecieron criterios de reparación universales pero que tenían que ser verificados antes de su ejecución. Una Reparación Profunda Uniforme, como se describió anteriormente, puede ser de una gran extensión en relación con el tamaño del elemento. Sin embargo, en este caso y para no recurrir a materiales, mano de obra y tiempo como es en el caso de la instalación de puntales de estabilización, se determinó que lo mejor era realizar las reparaciones en secciones de $1.5 \mathrm{~m}$ para no comprometer la estabilidad estructural. A continuación, se presenta el ejemplo de una columna tipo rectangular. La columna tipo (véase Figura 5), presentaba una sección transversal de 30x35 cm y con ayuda de las pruebas mecánicas realizadas se pudo obtener la resistencia promedio del concreto la cual se encuentra superior a los $200 \mathrm{~kg} / \mathrm{cm}^{2}$. Lo anterior nos permite entender que la columna es capaz de soportar una carga axial de $21,000 \mathrm{~kg}$. Ahora bien, si escarificamos el concreto en mal estado, y reparamos más allá del refuerzo, recordando que la reparación profunda uniforme 
es la que se da más allá de los $3 \mathrm{~cm}$ o detrás del refuerzo, la nueva geometría del elemento es de $25 \times 30 \mathrm{~cm}$ y con una resistencia por encima de $\operatorname{los} 200 \mathrm{~kg} / \mathrm{cm}^{2}$, podemos determinar que la estructura, aún sin una sección de concreto, es capaz de soportar $15,000 \mathrm{~kg}$. Si al diseño del elemento original le quitáramos un factor de seguridad de 1.5 obtendremos una resistencia de $14,000 \mathrm{~kg}$ por lo que el elemento escarificado aún presenta un margen de trabajo tolerable y seguro.

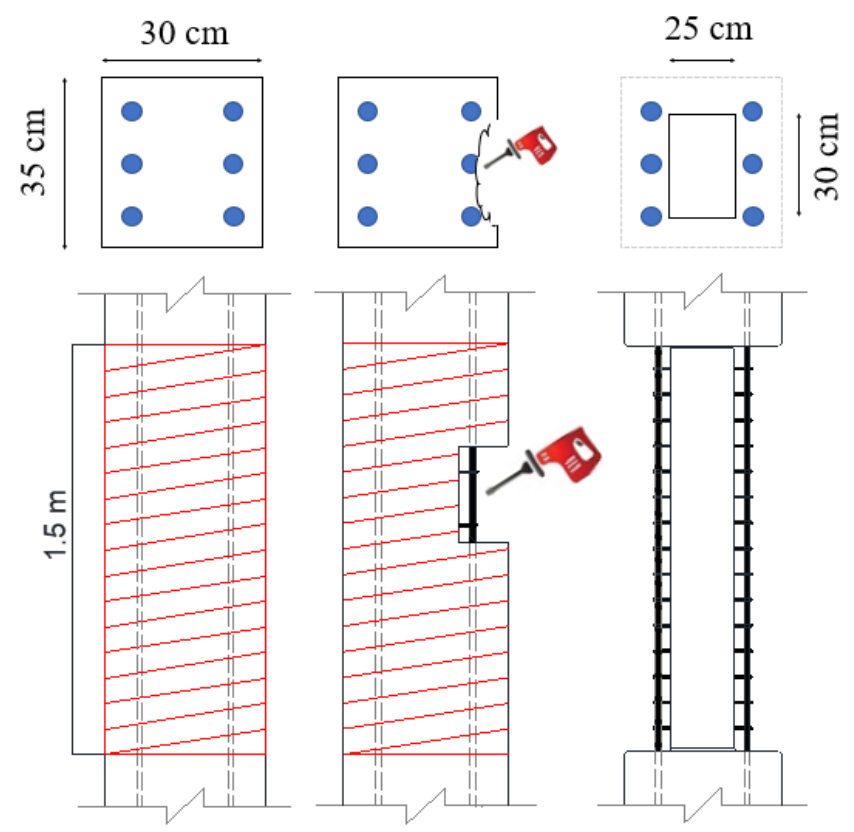

Figura. 5. Criterio de delimitación y escarificación de reparaciones

Para el proceso de reparación, se realizó una guía la cual pudiera ser seguida con facilidad por los obreros y que a su vez fuera repetible en varios elementos, en la Figura 6 puede observarse este proceso.

Escarificación mecánica para eliminar el concreto en mal estado y descubrir el acero de refuerzo

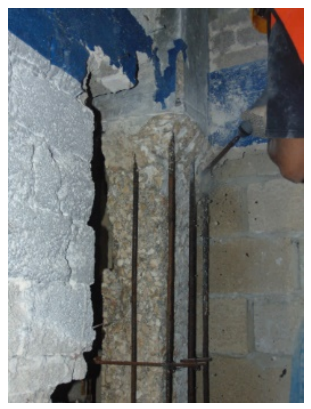

Medición del diámetro del acero de refuerzo, si la pérdida de diámetro era mayor a la permisible, el acero era completado o sustituido. En algunos casos, se colocó acero de refuerzo transversal completo por el desgaste excesivo

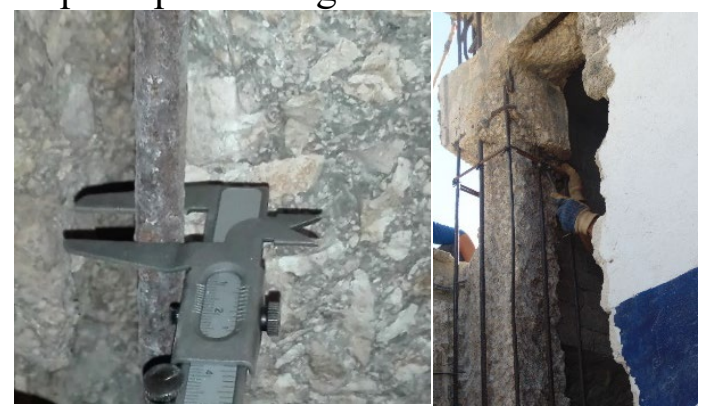


Se perforaron agujeros para la colocación del nuevo acero que sustituyera al corroído. Para su correcto anclaje, se inyectó una resina epóxica de alto rendimiento para altas cargas

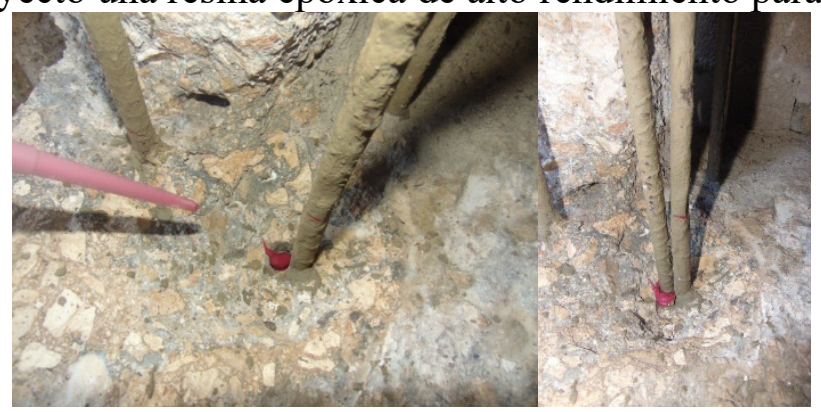

Antes de realizar el desplante, la base de todos los elementos fue recubierta con un impermeabilizante asfáltico alrededor del suelo para evitar alguna penetración de humedad o agentes corrosivos.

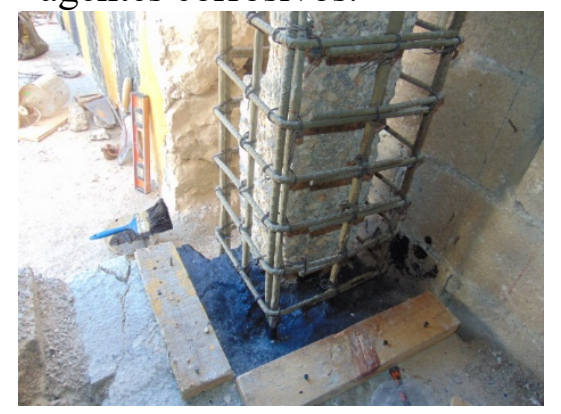

Por la experiencia previa de la inspección, se recomendó aplicar al acero un convertidor e inhibidor de óxido

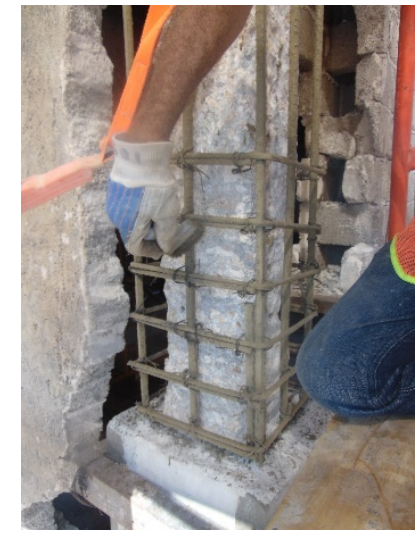

Previo al colado, se aplicó un puente de adherencia para asegurar la homogenización del concreto nuevo con el viejo, éste también tiene la función de sellador.

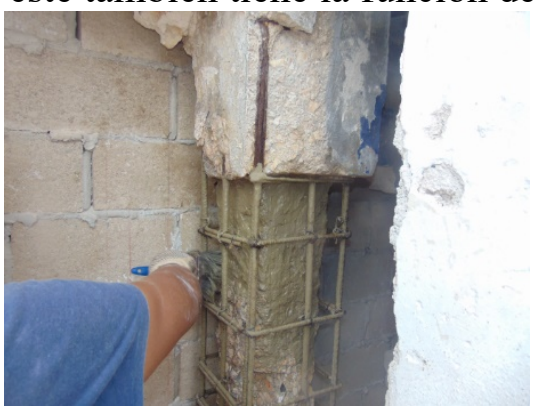


Posterior al colado y después del descimbrado, se aplicó una membrana de curado al concreto para asegurar la durabilidad.

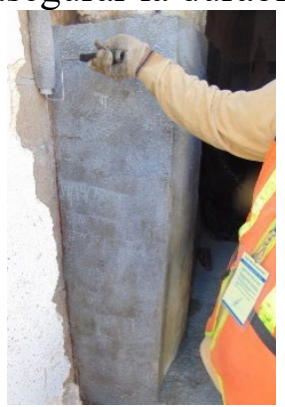

Figura. 6. Proceso de reparación profunda uniforme

\section{CONCLUSIÓN}

El presente trabajo tuvo como fin el presentar la rehabilitación de una estructura de concreto reforzado (véase Figura 7) de 70 años, la cual se encontraba en estado de abandono y se había proyectado la demolición total del mismo. Por mandamiento de las autoridades, se realizó un estudio de durabilidad que arrojó como resultado cambiar el proyecto de demolición a uno de restauración. Para realizar esta restauración, fue necesario consultar distintas fuentes didácticas como experiencias previas que arrojaron el realizar un manual práctico para los obreros. Este manual se basó en criterios de durabilidad, así como en especificaciones de diseño que permitan trabajar con seguridad. El uso de la teoría para la realización del diseño de una estructura es importante a lo largo de toda la vida de servicio de esta. Como describimos en este trabajo, el factor de seguridad tuvo un papel importante en la determinación del mecanismo de reparación que trajo consigo un importante ahorro en recursos y tiempo. Con estos procedimientos de reparación se proyecta tener una estructura durable que sea capaz de cumplir con la vida de servicio establecida con un mantenimiento preventivo

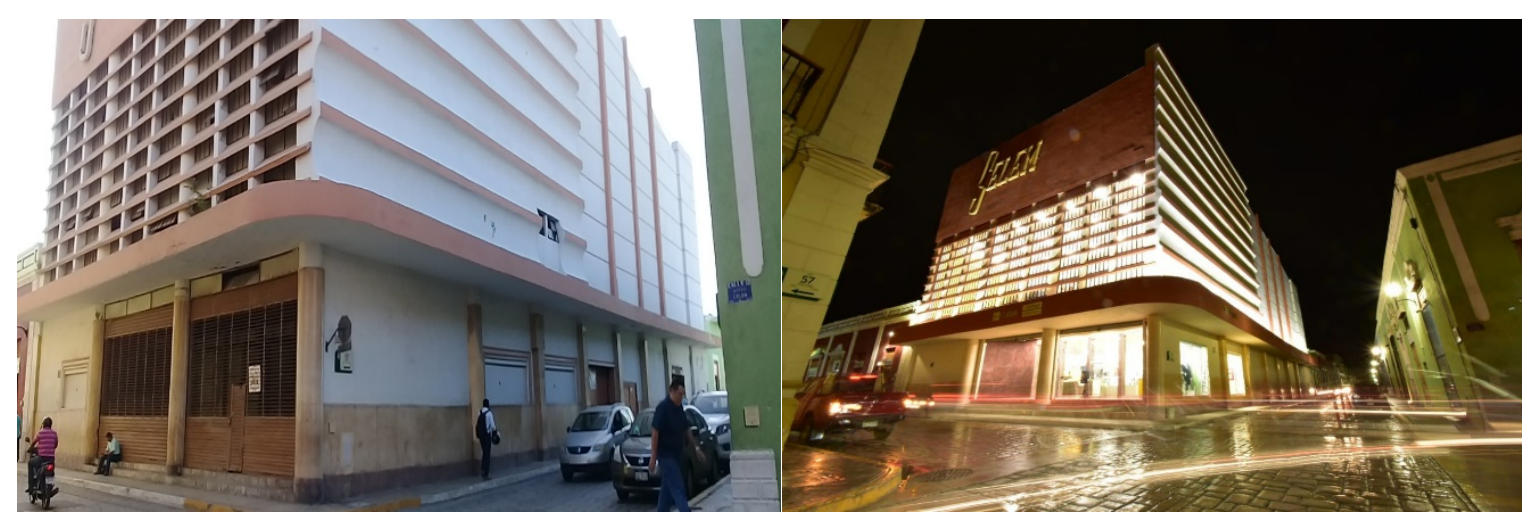

Figura. 7. Antes y después de la intervención

\section{AGRADECIMIENTOS}

Los autores agradecen el apoyo de Coppel SA de CV, la UACAM, Cinvestav, INAH e INBA. J.A. Briceño-Mena agradece la beca de doctorado de Conacyt. 


\section{REFERENCIAS}

[1] NMX-C-505-ONNCCE, "Industria de la Construcción - Inspección Preliminar de Daños en Estructuras de Concreto Hidráulico con Criterios de Durabilidad - Procedimientos," 2016.

[2] NMX-C-520-ONNCCE, "Industria de la Construcción-Inspección Detallada de Daños en Estructuras de Concreto Hidráulico con Criterios de Durabilidad-Procedimientos," 2015.

[3] J. A. Briceño-Mena, M. G. Balancán-Zapata, P. Castro-Borges, and T. Pérez-lópez, "Extending the Service Life of a Historical Concrete Building," Mater. Perform., pp. 1-8, 2021.

[4] NMX-C-530-ONNCCE, "Industria de la construcción - Durabilidad - Norma general de durabilidad de estructuras de concreto reforzado - Criterios y Especificaciones," 2017.

[5] P. Helene and F. Pereira, Manual de rehabilitación de estructuras de hormigón reparación, refuerzo y protección. Red Rehabilitar.

[6] A. Virginia et al., "Aplicación de la teoría de seguridad al diseño de cimentaciones en arenas . Chequeo de linealidad Application of the safety theory to the design of foundations in sands . Checkup of the linear limit," vol. 22, pp. 81-88, 2007.

[7] R. R. C. and P. H. O., Diseño Estructural, vol. 7. 2020. 